\title{
Cluster properties and particle production in Poincaré invariant quantum mechanics
}

\author{
W. N. Polyzou ${ }^{1, a}$ \\ Department of Physics and Astronomy, The University of Iowa, Iowa City, IA 52242
}

\begin{abstract}
I outline the construction of exactly Poincaré invariant quantum models that satisfy cluster separability but do not conserve particle number.
\end{abstract}

\section{Introduction}

Few-body physics has been transformational in terms of how we understand low-energy nuclear physics. The success is largely due to the existence of uncoupled few-body problems that are directly related to experiment and cluster properties that relate the few and many-body Hamiltonians. Thus, nucleon-nucleon interactions are fine tuned by comparing cross sections calculated using numerically exact solutions of the Lippmann-Schwinger equation to experimental nucleon-nucleon cross sections. Cluster properties fix how these nucleon-nucleon interactions appear in $\mathrm{N}>2$-body Hamiltonians. Small corrections due to threebody interactions can also be fine tuned by comparing numerically exact solutions of the Faddeev equations with experiment. Cluster properties again fix how the two and three-body interactions appear in the $\mathrm{N}>3$-body Hamiltonians. The saturation of nuclear binding energies suggests that at nuclear densities two, three and possibly four-body interactions are sufficient to construct Hamiltonians that provide an accurate description of most nuclei. The level of success achieved in low-energy nuclear physics has not been duplicated for energy scales above the threshold for the production of pions.

There are a number of reasons for the increased difficulty:

For energies approaching or above the GeV scale the Poincaré group must be a symmetry of the theory so calculations in the laboratory and center of momentum frame are consistent. Cluster properties become more difficult to satisfy in Poincaré invariant models. While cluster properties can be satisfied for fixed number of particles[1][2], there is no known systematic treatment of cluster properties for few-body systems where particle number is not conserved.

2.) A consistent treatment of particle production also requires a Poincaré symmetric treatment. Particle production violates Galilean invariance; momentum conservation

\footnotetext{
a e-mail: polyzou@uiowa.edu
}

cannot be simultaneously satisfied in two frames related by Galilean boosts.

3.) There is no few-body problem directly related to experiment. Even the one-nucleon problem involves an infinite number of bare pions. This makes it difficult to construct phenomenological few-body interactions by comparing numerically exact calculations to experiment.

4.)

Even if one can satisfy cluster properties, asymptotically separated clusters still involve infinite numbers of bare particle degrees of freedom.

The few-GeV scale is an important energy scale. It is the scale where it is possible to study the sensitivity of the dynamics to sub-nucleon degrees of freedom. Also, even though the physics involves an infinite number of bare particle degrees of freedom, we expect that the dynamics should be dominated by a finite number of suitably chosen degrees of freedom; it is hard to believe that a small change in energy would require the number of degrees of freedom needed to describe nucleon-nucleon scattering to suddenly jump from two to infinity.

In this talk I discuss a strategy to address all of the difficulties discussed above, extending the few-body program so it can be used to make realistic models of systems with invariant energy above the pion-production threshold that preserve the properties that have made few-body methods transformational below the pion-production threshold. The physics motivation for doing this is to extend the success of few-body methods to energy scales where sub-nucleon degrees of freedom become relevant. This is also relevant because it is now possible to solve relativistic Faddeev equations at the GeV scale [3][4][5].

To treat this problem it is useful to consider the structure of low-energy few-body models. In principle, from the point of view of perturbative field theory, even low-energy nucleon-nucleon scattering involves an infinite number of bare meson degrees of freedom. Physical nucleons can also be understood as bare nucleons with a cloud of virtual mesons. However, in low-energy nuclear physics one never deals with the bare nucleon degrees of freedom; instead the relevant degrees of freedom are taken as physical nucleons. Their masses are not calculated, they are measured. Even 
though the structure of the nucleons cannot be calculated, it can still be probed using scattering experiments. For example, electron proton-scattering suggests that the proton has a non-trivial electromagnetic structure. The fundamental vertex involving bare mesons and nucleons is replaced by a phenomenological nucleon-nucleon interaction between physical nucleons. While the Lippmann-Schwinger equation with realistic nucleon-nucleon interactions can be solved at all energies, it is only designed to be taken seriously for energies where the dominant degrees of freedom are two physical nucleons. This means that the number of relevant degrees of freedom is dictated by the energy scale of interest. All of this can even be done relativistically using Poincaré symmetric versions of the two-nucleon problem.

The observations in the previous paragraph suggest how to proceed. Building on the what was successful in the lowenergy case, we formulate the theory using only physicalparticle degrees of freedom. Thus, nucleons, deuterons, and pions will all be taken as physical particles. Since physical particles have no-self interactions, a theory formulated in terms of physical particle degrees of freedom cannot have elementary vertices, otherwise there would be mass renormalizations. Interactions must be short-ranged, involving two or more initial and two or more final particles. The simplest interaction that changes particle number is a shortranged $2 \leftrightarrow 3$ interaction. Even if the theory is formulated using physical particle degrees of freedom, if there is real particle production, the theory still involves an infinite number of degrees of freedom. To have few-body problems directly constrained by experiment it is necessary to replace a single theory that is applicable at all energy scales by an ordered sequence of effective theories with successively more degrees of freedom that are relevant over different energy scales. When a system of physical particles is broken up into asymptotically separated subsystems, the subsystems will involve lower-energy scales. Cluster properties can be realized if these lower energy sub-systems are constrained by the lower-energy effective theories.

In what follows I use the example of nucleon-nucleon scattering between the one and two pion-production threshold to illustrate the structure and construction of such a theory.

\section{Hilbert space}

Minimally, the model Hilbert space must have enough structure so that it is possible to compute probability amplitudes for any experiment of interest. For experiments at a fixed energy scale, it is possible to measure the momenta and spin projections of every particle that can appear in an initial or final state. This motivates the choice of model Hilbert space as the direct sum of tensor products of single physical-particle Hilbert spaces, where the particle content corresponds to the particles that can be experimentally observed at the given energy scale. This space has enough degrees of freedom to describe the results of any experiment that can be performed at the given energy scale.
Complete experiments on isolated physical particles measure their mass, linear momentum, spin, and spin polarization with respect to some axis in a given frame. A suitable representation of the one-particle Hilbert space, $\mathcal{H}_{1}$, is the space of square integrable functions, $\psi(\mathbf{p}, \mu)$, of these observables

$$
\begin{gathered}
\psi(\mathbf{p}, \mu)=\langle(m, j) \mathbf{p}, \mu \mid \psi\rangle \\
\langle\psi \mid \psi\rangle=\int \sum_{\mu=-j}^{j}|\psi(\mathbf{p}, \mu)|^{2} d \mathbf{p}<\infty
\end{gathered}
$$

where $(m, j)$ are the physical mass and spin of the particle.

The formulation of cluster properties works best when the theory is formulated in terms of tensor products. In order to preserve the tensor product structure in models with particle production, it is useful to replace the single particle Hilbert space by the direct sum of a single particle Hilbert space and a zero dimensional no-particle space:

$$
\mathcal{H}_{1} \rightarrow \mathcal{H}_{1} \oplus\{0\}
$$

These doublet spaces were first introduced by Sokolov[1]. The tensor product of doublet spaces can be decomposed as a direct sum of tensor products. For example, if the system has particles of type $b, c$, and $d$, the tensor product of the three doublet spaces can be expanded into a direct sum of subspaces with all particle contents involving particles $b, c$, and $d$.

$$
\begin{gathered}
\left(\mathcal{H}_{b} \oplus\{0\}\right) \otimes\left(\mathcal{H}_{c} \oplus\{0\}\right) \otimes\left(\mathcal{H}_{d} \oplus\{0\}\right)= \\
\left(\mathcal{H}_{b} \otimes \mathcal{H}_{c} \otimes \mathcal{H}_{d}\right) \oplus\left(\mathcal{H}_{b} \otimes \mathcal{H}_{c}\right) \oplus\left(\mathcal{H}_{c} \otimes \mathcal{H}_{d}\right) \oplus \\
\left(\mathcal{H}_{d} \otimes \mathcal{H}_{b}\right) \oplus \mathcal{H}_{b} \oplus \mathcal{H}_{c} \oplus \mathcal{H}_{d} \oplus\{0\} .
\end{gathered}
$$

In specific models some of these combinations do not appear because of super-selection rules, energy considerations, or properties of the interactions. In what follows, we construct our models assuming that the particles are initially distinguishible, and then treat the particle identity when computing the cross sections.

The single-particle spaces are also irreducible representation spaces for the Poincaré group. The unitary irreducible representation, $U_{1}(\Lambda, a)$, of the Poincaré group on the single particle Hilbert space $\mathcal{H}_{1}$ is

$$
\begin{gathered}
\left\langle(m, j) \mathbf{p}, \mu\left|U_{1}(\Lambda, a)\right| \psi\right\rangle= \\
\int \sum_{\mu^{\prime}=-j}^{j}\left\langle(m, j) \mathbf{p}, \mu\left|U_{1}(\Lambda, a)\right|(m, j) \mathbf{p}^{\prime}, \mu^{\prime}\right\rangle d \mathbf{p}^{\prime} \times \\
\left\langle(m, j) \mathbf{p}^{\prime}, \mu^{\prime} \mid \psi\right\rangle= \\
\int \sum_{\mu^{\prime}=-j}^{j} \mathcal{D}_{\mathbf{p}, \mu ; \mathbf{p}^{\prime}, \mu^{\prime}}^{m j}[\Lambda, a] d \mathbf{p}^{\prime} \psi\left(\mathbf{p}^{\prime}, \mu^{\prime}\right)
\end{gathered}
$$

where the Poincaré group Wigner function is

$$
\mathcal{D}_{\mathbf{p}, \mu ; \mathbf{p}^{\prime}, \mu^{\prime}}^{m j}[\Lambda, a]:=\left\langle(m, j) \mathbf{p}, \mu\left|U_{1}(\Lambda, a)\right|(m, j) \mathbf{p}^{\prime}, \mu^{\prime}\right\rangle=
$$




$$
e^{i p \cdot a} \sqrt{\frac{\omega_{m}(\mathbf{p})}{\omega_{m}\left(\mathbf{p}^{\prime}\right)}} \delta\left(\mathbf{p}-\Lambda p^{\prime}\right) D_{\mu \mu^{\prime}}^{i}\left[B^{-1}(p / m) \Lambda B\left(p^{\prime}, m\right)\right]
$$

with

$$
\omega_{m}(\mathbf{p})=\sqrt{\mathbf{p}^{2}+m^{2}}
$$

and $B(p / m)$ is a Lorentz boost

$$
B(p / m)(m, 0,0,0)=p
$$

that depends on the choice of spin polarization observable (helicity, canonical spin, null-plane spin). The quantity $B^{-1}(p / m) \Lambda B\left(p^{\prime} / m\right)$ is a Wigner rotation.

The direct sum of tensor products of these single-particle unitary irreducible representations, where the representation acts like the identity on the zero dimensional noparticle state, defines the non-interacting representation, $U_{0}(\Lambda, a)$, of the Poincaré group on the model Hilbert space. On the tensor product of the three doublet spaces (4) it has the form

$$
\begin{gathered}
U_{0}(\Lambda, a)=\left[U_{a}(\Lambda, a) \otimes U_{b}(\Lambda, a) \otimes U_{c}(\Lambda, a)\right] \oplus \\
{\left[U_{a}(\Lambda, a) \otimes U_{b}(\Lambda, a)\right] \oplus\left[U_{b}(\Lambda, a) \otimes U_{c}(\Lambda, a)\right] \oplus} \\
{\left[U_{c}(\Lambda, a) \otimes U_{a}(\Lambda, a)\right] \oplus U_{a}(\Lambda, a) \oplus} \\
U_{b}(\Lambda, a) \oplus U_{c}(\Lambda, a) \oplus I .
\end{gathered}
$$

\section{Nucleon-nucleon scattering model}

Consider nucleon-nucleon scattering for invariant energy between $2 m_{N}+m_{\pi}$ and $2 m_{N}+2 m_{\pi}$. In this energy range the possible baryon number two states are $(N N),(N N \pi),(D \pi)$, $(D \pi \pi)$. Using Sokolov's doublet formalism and treating the nucleons and pions as distinguishible, the model Hilbert space is the following direct sum of tensor products of two and three particle spaces:

$$
\begin{gathered}
\mathcal{H}=\mathcal{H}_{N_{1} N_{2}} \oplus \mathcal{H}_{N_{1} N_{2} \pi_{1}} \oplus \mathcal{H}_{N_{1} N_{2} \pi_{2}} \oplus \\
\mathcal{H}_{D \pi_{1}} \oplus \mathcal{H}_{D \pi_{2}} \oplus \mathcal{H}_{D \pi_{1} \pi_{2}} .
\end{gathered}
$$

Vectors in this space are represented by six-component wave functions:

$$
\Psi(\cdots)=\left(\begin{array}{c}
\psi_{N N}\left(\mathbf{p}_{N}, \mu_{N}, \mathbf{p}_{N^{\prime}}, \mu_{N^{\prime}}\right) \\
\psi_{D \pi}\left(\mathbf{p}_{D}, \mu_{D}, \mathbf{p}_{\pi}\right) \\
\psi_{D \pi^{\prime}}\left(\mathbf{p}_{D}, \mu_{D}, \mathbf{p}_{\pi^{\prime}}\right) \\
\psi_{N N \pi}\left(\mathbf{p}_{N}, \mu_{N}, \mathbf{p}_{N^{\prime}}, \mu_{N^{\prime}}, \mathbf{p}_{\pi}\right) \\
\psi_{N N \pi^{\prime}}\left(\mathbf{p}_{N}, \mu_{N}, \mathbf{p}_{N^{\prime}}, \mu_{N^{\prime}}, \mathbf{p}_{\pi^{\prime}}\right) \\
\psi_{D \pi \pi}\left(\mathbf{p}_{D}, \mu_{P}, \mathbf{p}_{\pi}, \mathbf{p}_{\pi^{\prime}}\right)
\end{array}\right) .
$$

The kinematic unitary representation of the Poincaré group is

$$
\begin{gathered}
U_{0}(\Lambda, a)=\left[U_{N}(\Lambda, a) \otimes U_{N^{\prime}}(\Lambda, a)\right] \oplus\left[U_{D}(\Lambda, a) \otimes U_{\pi}(\Lambda, a)\right] \oplus \\
{\left[U_{D}(\Lambda, a) \otimes U_{\pi^{\prime}}(\Lambda, a)\right] \otimes\left[U_{N}(\Lambda, a) \otimes U_{N^{\prime}}(\Lambda, a) \otimes U_{\pi}(\Lambda, a)\right] \oplus} \\
{\left[U_{N}(\Lambda, a) \otimes U_{N^{\prime}}(\Lambda, a) \otimes U_{\pi^{\prime}}(\Lambda, a)\right] \oplus} \\
{\left[U_{D}(\Lambda, a) \otimes U_{\pi}(\Lambda, a) \otimes U_{\pi^{\prime}}(\Lambda, a)\right] .}
\end{gathered}
$$

\section{Dynamics}

In a quantum mechanical model a Poincaré symmetry is implemented by a unitary representation of the Poincaré group[6]. The representation is necessarily dynamical[7].

The free-particle representation, $U_{0}(\Lambda, a)$, is reducible on the model Hilbert space $\mathcal{H}$. Poincaré group ClebschGordan coefficients [8][9][10] can be used to decompose the Hilbert space into a direct integral of invariant subspaces on which $U_{0}(\Lambda, a)$ acts irreducibly. The ClebschGordan coefficients have the form

$$
\begin{gathered}
\langle(12) \mid 3\rangle= \\
\left\langle\left(m_{1}, j_{1}\right) \mathbf{p}_{1}, \mu_{1}\left(m_{1}, j_{2}\right) \mathbf{p}_{2}, \mu_{2} \mid\left(\mathbf{k}^{2}, j_{3}\right) \mathbf{p}_{3}, \mu_{3}, d\right\rangle
\end{gathered}
$$

where $\mathbf{k}^{2}$ is a more convenient label for the invariant mass $m_{3}$ of the combined system

$$
m_{3}=\sqrt{m_{1}^{2}+\mathbf{k}^{2}}+\sqrt{m_{2}^{2}+\mathbf{k}^{2}}
$$

In what follows we use $m_{3}$ and $\mathbf{k}^{2}=k^{2}$ interchangeably. Multiple copies of invariant subspaces with the same mass and spin are separated by invariant degeneracy parameters $d$. The parameters $d$ are related to the squares of spin $s^{2}$ and orbital angular momentum $l^{2}$ of the 12-pair. Three particle irreducible representations can be constructed by successive pairwise coupling.

The irreducible basis states are labeled by the same quantities that are used to label the single-particle states; linear momentum, mass, spin, spin projection, as well as a number of Poincaré invariant degeneracy parameters which we label by $d_{n}$, where $n \in\left\{N N, N N \pi, N N \pi^{\prime}, D \pi, D \pi^{\prime}, D \pi \pi\right\}$. These eigenstates are complete on the model Hilbert space. A basis of non-interacting Poincaré irreducible states consists of the generalized vectors

$$
\left|(m, j) \mathbf{p}, \mu, d_{n}, n\right\rangle=\left(\begin{array}{c}
0 \\
\vdots \\
\left|(m, j) \mathbf{p}, \mu, d_{n}\right\rangle \\
\vdots \\
0
\end{array}\right) .
$$

The non-interacting invariant mass operator, $M_{0}$, is the mass Casimir operator of $U_{0}(\Lambda, a)$. It has a continuous spectrum and is a multiplication operator in the representation (15). Interactions of the form [11]:

$$
\begin{aligned}
& \left\langle\left(m^{\prime}, j^{\prime}\right) \mathbf{p}^{\prime}, \mu^{\prime}, d_{n^{\prime}}^{\prime}, n^{\prime}|V|(m, j) \mathbf{p}, \mu, d_{n}, n\right\rangle= \\
& \delta\left(\mathbf{p}^{\prime}-\mathbf{p}\right) \delta_{j^{\prime} j} \delta_{\mu^{\prime} \mu}\left\langle m^{\prime}, d_{n^{\prime}}^{\prime}, n^{\prime}\left\|V^{j}\right\| m, d_{n}, n\right\rangle
\end{aligned}
$$

are added to the non-interacting mass operator, $M_{0}$, to construct a dynamical mass operator:

$$
M=M_{0}+V
$$

Simultaneous eigenstates $|(\lambda, j) \mathbf{p}, \mu, d\rangle$ of $M, j, \mathbf{p}, \mu$ are complete. The dynamical problem is to solve the eigenvalue problem

$$
\left(M_{0}+V\right)|(\lambda, j) \mathbf{p}, \mu, d\rangle=\lambda|(\lambda, j) \mathbf{p}, \mu, d\rangle
$$


in the non-interacting irreducible basis. The eigenfunctions in the non-interacting irreducible representation can be expressed in terms of wave functions $\Psi_{\lambda, j, d}\left(m^{\prime}, d_{n^{\prime}}^{\prime}, n^{\prime}\right)$ :

$$
\begin{gathered}
\left\langle\left(m^{\prime}, j^{\prime}\right) \mathbf{p}^{\prime}, \mu^{\prime}, d_{n^{\prime}}^{\prime}, n^{\prime} \mid(\lambda, j) \mathbf{p}, \mu, d\right\rangle= \\
\delta\left(\mathbf{p}^{\prime}-\mathbf{p}\right) \delta_{j^{\prime} j} \delta_{\mu^{\prime} \mu} \Psi_{\lambda, j, d}\left(m^{\prime}, d_{n^{\prime}}^{\prime}, n^{\prime}\right) .
\end{gathered}
$$

There is a natural dynamical unitary representation of the Poincaré group defined on these eigenstates by

$$
\begin{gathered}
\left\langle\left(m^{\prime}, j^{\prime}\right) \mathbf{p}^{\prime}, \mu^{\prime}, d_{n^{\prime}}^{\prime}, n^{\prime}|U(\Lambda, a)|(\lambda, j) \mathbf{p}, \mu, d\right\rangle= \\
\Psi_{\lambda, j, d}\left(m^{\prime}, d_{n^{\prime}}^{\prime} n^{\prime}\right) \mathcal{D}_{\mathbf{p}, \mu ; \mathbf{p}^{\prime}, \mu^{\prime}}^{\lambda j}[\Lambda, a]
\end{gathered}
$$

where $\lambda$ the eigenvalue of $M$. Completeness of the eigenstates ensures that this representation is defined on any state. The dynamics enters through the appearance of the mass eigenvalue, $\lambda$, in the Poincaré group Wigner function $\mathcal{D}_{\mathbf{p}, \mu ; \mathbf{p}^{\prime}, \mu^{\prime}}^{\lambda j}[\Lambda, a]$.

This construction can be done for any finite number of degree of freedom system. What is relevant in this construction is that the spin $j$ in the dynamical model is the same as the spin $j$ in the non-interacting model. We refer to this construction of dynamical representation of the Poincaré group as the generalized Bakamjian-Thomas[11] construction.

Three different two-body models are needed as input to the full dynamical model. These two-body models are a $\pi-N$ model, a $\pi-\pi$ model and a coupled channel $N N \leftrightarrow$ $D \pi$ model. In what follows we replace the invariant mass $m_{3}$ by the relative momentum variable $k=\sqrt{\mathbf{k}^{2}}$. For the coupled channel model the non-interacting invariant mass operator has the form

$$
M_{0}:=\left(\begin{array}{cc}
2 \sqrt{k_{N N}^{2}+m_{N}^{2}} & 0 \\
0 & \sqrt{k_{D \pi}^{2}+m_{D}^{2}}+\sqrt{k_{D \pi}^{2}+m_{\pi}^{2}}
\end{array}\right) .
$$

The interaction $V$ is defined by the kernel in the irreducible two-body variables

$$
\begin{gathered}
\delta\left(\mathbf{p}^{\prime}-\mathbf{p}\right) \delta_{j^{\prime} j} \delta_{\mu^{\prime} \mu} \times \\
\left(\begin{array}{c}
\left\langle k_{n n}^{\prime}, l^{\prime}, s^{\prime}\left|v_{N N ; N N}^{j}\right| k_{n n}, l, s\right\rangle\left\langle k_{n n}^{\prime}, l^{\prime}, s^{\prime}\left|v_{N N ; D \pi}^{j}\right| k_{D \pi}, l, 1\right\rangle \\
\left\langle k_{D \pi}^{\prime}, l^{\prime}, 1\left|v_{D \pi ; N N}^{j}\right| k_{n n}, l, s\right\rangle\left\langle k_{D \pi}^{\prime}, l^{\prime}, 1\left|v_{D \pi ; D \pi}^{j}\right| k_{D \pi}, l, 1\right\rangle
\end{array}\right) .
\end{gathered}
$$

The dynamical mass operator on $\mathcal{H}_{N N} \oplus \mathcal{H}_{D \pi}$ is $M=M_{0}+$ $V$, where $V$ is the interaction defined by the kernel (22). Simultaneous eigenstate of $M, j, \mathbf{p}$, and $\hat{\mathbf{z}} \cdot \mathbf{j}$ from a complete set of basis vectors that transform irreducibly with respect to a dynamical representation $U_{N N-D \pi}(\Lambda, a)$

$$
\begin{gathered}
\left\langle\left(\lambda^{\prime}, j^{\prime}\right) \mathbf{p}^{\prime}, \mu^{\prime}, d^{\prime}\left|U_{N N-D \pi}(\Lambda, a)\right|(\lambda, j) \mathbf{p}, \mu, d\right\rangle= \\
\delta_{j^{\prime} j} \delta\left(\lambda^{\prime}-\lambda\right) \delta_{d^{\prime} d} \mathcal{D}_{\mathbf{p}, \mu ; \mathbf{p}^{\prime}, \mu^{\prime}}^{\lambda j}[\Lambda, a]
\end{gathered}
$$

where $\lambda$ is the eigenvalue of the dynamical mass operator $M$. A similar construction can be done for the $\pi-\pi$ and $\pi-$ $N$ systems, resulting in the representations $U_{N N-\pi D}(\Lambda, a)$ on $\mathcal{H}_{N N} \oplus \mathcal{H}_{D \pi}, U_{N-\pi}(\Lambda, a)$ on $\mathcal{H}_{N \pi}$ and $U_{\pi \pi}(\Lambda, a)$ on $\mathcal{H}_{\pi \pi}$. These models are only required to fit scattering below the pion-production threshold. Because the bound states are treated as physical degrees of freedom, the spectrum of the mass operator in these two-body models is continuous.

\section{Algebraic cluster properties}

Cluster properties dictate how two-body interaction are embedded in the three-body sectors of the model Hilbert space. Cluster properties are normally implemented by translations. In this framework translations used to separate subsystems only operate on subspaces of the Hilbert space were the translations make sense; for example it makes no sense to separate nucleons on a subspace containing the deuteron. Translations that asymptotically separate subsystems ensure that the short-ranged interactions between particles in separated clusters vanish, however the difficulty in Poincare invariant quantum mechanics is that the translations can cause interactions that should not vanish to vanish in the cluster limit. This will not happen if result of simply turning-off interactions results in a tensor product of subsystem unitary representations of the Poincaré group. We call this type of clustering algebraic clustering and use it in what follows.

Consider the problem of nucleon-nucleon scattering for invariant energies between the one and two pion-production threshold. The model Hilbert has six orthogonal sectors with different particle contents. There are three three-body sectors, each having at least one pion. If we translate one of the particles away from an interacting pair in one of the three-body sectors, then the invariant energy remaining for the interacting pair is insufficient to produce another pion. Algebraic cluster properties requires that in this limit the Poincaré generators in this sector should become the sum of one-body generators corresponding to the spectator particle and dynamical two-body generators associated with an interacting two-body systems having insufficient invariant energy to create an additional pion. This is equivalent to the dynamical unitary representation of the Poincaré group becoming a tensor product. In each of these sectors there are three different two-body interactions that arise from cluster properties, depending on which particle is asymptotically separated.

These are the only constraints that cluster properties place on this model. The two-body sectors are relevant for invariant energies above the threshold for the production of a pion and in principle do not have to be related to the lower-energy two-body Hamiltonians.

The problem of two interacting particles and a spectator can be solved using two different methods. The first one is to construct the two-body unitary representation of the Poincaré group, $U_{N N-D \pi}(\Lambda, a), U_{N \pi}(\Lambda, a), U_{\pi \pi^{\prime}}(\Lambda, a)$ and then take the tensor product with the spectator representation

$$
\begin{gathered}
U_{N N-D \pi}(\Lambda, a) \otimes U_{\pi^{\prime}}(\Lambda, a), \\
U_{N \pi}(\Lambda, a) \otimes U_{N^{\prime}}(\Lambda, a), \\
U_{\pi \pi^{\prime}}(\Lambda, a) \otimes U_{D}(\Lambda, a) .
\end{gathered}
$$

The second method uses the standard construction where we first use Poincaré Clebsch Gordan coefficients to decompose the non-interacting three-body representations into a direct integral of non-interacting irreducible representations. Interactions of the from (16) are added to the noninteracting mass, which is then diagonalized to construct 


\section{$19^{\text {th }}$ International IUPAP Conference on Few-Body Problems in Physics}

$U(\Lambda, a)$. We refer to these two representations as the tensor product representation and the Bakamjian-Thomas representation respectively. I denote the Bakamjian-Thomas representations by

$$
\begin{gathered}
U_{N N-D \pi ; \pi^{\prime}}(\Lambda, a), \\
U_{N \pi ; N^{\prime}}(\Lambda, a), \\
U_{\pi \pi^{\prime} ; D}(\Lambda, a) .
\end{gathered}
$$

These operators do not act on the full Hilbert space. They act on a subspace of the full Hilbert space. They can be extended to operators on the full Hilbert space by extending them to be the identity on the orthogonal complement of the subspace.

The kernel of the two-body interaction in the two-body kinematic irreducible representation (16) depends on the kinematic two-body relative momentum and kinematicallyinvariant two-body degeneracy parameters. By coupling irreducible representations in the appropriate order, the same variables also appear in the three-body kinematic irreducible basis. Using the same kernel, with different delta functions, it is easy to show that the tensor product and BakamjianThomas representations of the dynamics of two interacting particles and a spectator give the same $2+1 \mathrm{~S}$-matrix. This follows because the delta functions that multiply the interaction also appear in the $S$-matrix, and they become equivalent when the $S$-matrix is evaluated on-shell. While the $2+1$ unitary representations of the Poincare group are not-equivalent, a theorem of Ekstein [12] implies that each representation is related by a unitary transformation, $W$, that also preserves the $S$ matrix.

Thus these two representations are related by

$$
\begin{gathered}
W_{N N-D \pi ; \pi^{\prime}} U_{N N-D \pi ; \pi^{\prime}}(\Lambda, a) W_{N N-D \pi ; \pi^{\prime}}^{\dagger}= \\
U_{N N-D \pi}(\Lambda, a) \otimes U_{\pi^{\prime}}(\lambda, a) \\
W_{N \pi ; N^{\prime}} U_{N \pi ; N^{\prime}}(\Lambda, a) W_{N \pi ; N^{\prime}}^{\dagger}=U_{N \pi}(\Lambda, a) \otimes U_{N^{\prime}}(\Lambda, a) \\
W_{\pi \pi^{\prime} ; D} U_{\pi \pi^{\prime} ; D}(\Lambda, a) W_{\pi \pi^{\prime} ; D}^{\dagger}=U_{\pi \pi^{\prime}}(\Lambda, a) \otimes U_{D}(\Lambda, a) .
\end{gathered}
$$

Because the interactions in the Bakamjian-Thomas representation commute with the kinematic spin, they can be combined with the remaining short-ranged interactions in a manner that leads to an overall interaction of the form (16). The Bakamjian Thomas construction then be used to construct a dynamical representation of the Poincaré group on the model Hilbert space.

The unitary operators $W_{x}$ operate on a subspace of the Hilbert space. They can be extended to unitary operators on $\mathcal{H}$ by setting them equal to the identity on the orthogonal complement of the space on which they are defined.

\section{Construction of the dynamical mass operator}

The dynamical mass operator for our model in the Bakamjian-Thomas representation has three distinct types of contributions
The first is the mass operator $M_{0}$ for the non-interacting system

$$
M_{0}=\left(\begin{array}{cccccc}
M_{0 N N^{\prime}} & 0 & 0 & 0 & 0 & 0 \\
0 & M_{0 D \pi} & 0 & 0 & 0 & 0 \\
0 & 0 & M_{0 D \pi^{\prime}} & 0 & 0 & 0 \\
0 & 0 & 0 & M_{0 N N^{\prime} \pi} & 0 & 0 \\
0 & 0 & 0 & 0 & M_{0 N N^{\prime} \pi^{\prime}} & 0 \\
0 & 0 & 0 & 0 & 0 & M_{0 D \pi \pi^{\prime}}
\end{array}\right)
$$

where the non-zero entries in this matrix are the invariant masses of the systems of particles on each subspace. This is the limiting form of this mass operator when all interactions are switched off.

Next we add lower-energy two-body interactions in each of the three-particle sectors:

$$
V_{2}=\left(\begin{array}{cccccc}
0 & 0 & 0 & 0 & 0 & 0 \\
0 & 0 & 0 & 0 & 0 & 0 \\
0 & 0 & 0 & 0 & 0 & 0 \\
0 & 0 & 0 & v_{N N^{\prime}}+v_{N \pi}+v_{N^{\prime} \pi} & 0 & v_{N N ; \pi D} \\
0 & 0 & 0 & 0 & v_{N N^{\prime}}+v_{N \pi^{\prime}}+v_{N^{\prime} \pi^{\prime}} & v_{N N ; \pi^{\prime} D} \\
0 & 0 & 0 & v_{N N ; \pi D}^{\dagger} & v_{N N ; \pi^{\prime} D}^{\dagger} & v_{D \pi}+v_{D \pi^{\prime}}+v_{\pi \pi^{\prime}}
\end{array}\right) .
$$

These are the interactions that come from the two-body Bakamjian-Thomas representations. They are related to the tensor product representations by the unitary transformations $W$. For example:

$$
\begin{gathered}
\left(\begin{array}{cccccc}
0 & 0 & 0 & 0 & 0 & 0 \\
0 & 0 & 0 & 0 & 0 & 0 \\
0 & 0 & 0 & 0 & 0 & 0 \\
0 & 0 & 0 & v_{N N^{\prime}} & 0 & v_{N N ; \pi D} \\
0 & 0 & 0 & 0 & 0 & 0 \\
0 & 0 & 0 & v_{N N ; \pi D}^{\dagger} & 0 & v_{D \pi}
\end{array}\right)= \\
W_{N N \leftrightarrow D \pi^{\prime}}^{\dagger}\left(\begin{array}{cccccc}
0 & 0 & 0 & 0 & 0 & 0 \\
0 & 0 & 0 & 0 & 0 & 0 \\
0 & 0 & 0 & 0 & 0 & 0 \\
0 & 0 & 0 & m_{N N^{\prime} \otimes \pi} & 0 & m_{N N ; \pi^{\prime} D \otimes \pi} \\
0 & 0 & 0 & 0 & 0 & 0 \\
0 & 0 & 0 & m_{N N ; \pi^{\prime} D \otimes \pi}^{\dagger} 0 & m_{D \pi^{\prime} ; \otimes \pi}
\end{array}\right) W_{N N \leftrightarrow D \pi^{\prime}}^{\dagger}- \\
\left(\begin{array}{lllllc}
0 & 0 & 0 & 0 & 0 & 0 \\
0 & 0 & 0 & 0 & 0 & 0 \\
0 & 0 & 0 & 0 & 0 & 0 \\
0 & 0 & 0 & m_{0 N N^{\prime} \pi} & 0 & 0 \\
0 & 0 & 0 & 0 & 0 & 0 \\
0 & 0 & 0 & 0 & 0 & m_{0 D \pi^{\prime} \pi}
\end{array}\right) .
\end{gathered}
$$

The third class of operators, $V_{3}$ are fully connected operators. These are two-body interactions in the two-body sectors, three-body interactions in the three-body sectors, 2-2 interactions coupling different two-body sectors and $2-$ 3-body interactions coupling two and three-body sectors. All of the two-body interactions in $V_{3}$ describe physics above the pion-production threshold and thus do not have to be related to the interactions below the pion-production threshold. In addition to being connected, all of these interactions must have kernels of the form (16) in the noninteracting Poincaré irreducible representation. These connected operators do not contribute to the cluster limit associated with lower energy subsystems. 
In this way the operator $M_{B T}=M_{0}+V_{2}+V_{3}$ commutes with $j$ and commutes with and is independent of $\mathbf{p}$ and $\mathbf{j} \cdot \hat{\mathbf{z}}$. Simultaneous eigenstates of these operators are complete and transform irreducibly as mass $\lambda=$ eigenvalue of $M_{B T}$, spin $j$, irreducible representations of the Poincaré group. We denote the resulting unitary representation of the Poincaré group by $U_{B T}(\Lambda, a)$. When interactions between cluster $b$ and $c$ in one of the three-body sectors are turned off, $U_{B T}(\Lambda, a)$ fails to break up into a tensor product. Instead it becomes:

$$
U_{B T}(\Lambda, a) \rightarrow W_{b c}\left[\left(U_{b}(\Lambda, a) \otimes U_{c}(\Lambda, a)\right) \oplus \cdots\right] W_{b c}^{\dagger}
$$

This can be repaired defining a symmetric product of the $W$ operators

$$
W:=e^{\sum \ln W_{a}}
$$

where the sum runs over

$$
\begin{gathered}
a \in\left\{\left[(N N-D \pi) ; \pi^{\prime}\right],\left[\left(N N-D \pi^{\prime}\right) ; \pi\right],\left(\pi \pi^{\prime} ; D\right),\left(D \pi ; \pi^{\prime}\right),\right. \\
\left.\left(D \pi^{\prime} ; \pi\right),\left(N \pi ; N^{\prime}\right),\left(N^{\prime} \pi ; N\right),\left(N \pi^{\prime} ; N^{\prime}\right),\left(N^{\prime} \pi^{\prime} ; N\right)\right\} .
\end{gathered}
$$

This operator is designed so

$$
\begin{gathered}
U(\Lambda, a):= \\
W^{\dagger} U_{B T}(\Lambda, a) W \rightarrow\left[\left(U_{b}(\Lambda, a) \otimes U_{c}(\Lambda, a)\right) \oplus \cdots\right]
\end{gathered}
$$

when the interactions between particles in cluster $b$ and $c$ are turned off. This shows that it satisfies algebraic cluster properties. Because $W$ is unitary, $U(\Lambda, a):=$ $W^{\dagger} U_{B T}(\Lambda, a) W$, is also a dynamical representation of the Poincaré group.

This completes the construction of the dynamics for the two-nucleon system of energies between the one and twopion production threshold. Thus we have a two-particle model that describes nucleon-nucleon scattering below the one-pion-production threshold, and we have a second model that described nucleon-nucleon scattering for invariant energies between the one and two-pion-production thresholds. While the models are uncoupled, interactions from the lower energy two-body model appear in the threebody sector of the higher-energy model. Both models are exactly Poincaré invariant with interactions that can be directly constrained by few-body experiments.

This general construction can be repeated inductively at higher energy scales. By using the doublets the entire inductive construction used in the fixed number of particle case [2] can be generalized to this setting.

\section{Outlook}

This work demonstrates that it is possible to overcome all of the difficulties in needed to extend the successful lowenergy few-body program to higher energy scales. In this formalism the independent degrees of freedom are taken as physical particles. This also applies to bound states. This is not a problem in principle, but it does mean that much of the work done in constructing realistic few-body interactions needs to be repeated in this setting. Another new feature is that the many-body interactions of the non-relativistic theory are replaced by both many-body interactions and hard (high-energy) two-body interactions. At each successive energy scale it is necessary to introduce new hard twobody interactions. In most cases these interactions appear in the entrance channel and thus cannot be ignored. An independent method for constructing these hard interaction would prove to be very useful in this framework. Still, a good part of the high-energy dynamics is strongly constrained by the few-body dynamics through cluster properties.

\section{Acknowledgement}

This work was performed in part under the auspices of the U. S. Department of Energy, Office of Nuclear Physics, contract No. DE-FG02-86ER40286 with the University of Iowa.

\section{References}

1. S. N. Sokolov, Dokl. Akad. Nauk USSR 233(1977)575.

2. F. Coester and W.N. Polyzou, Phys. Rev. D26(1982)1348.

3. T. Lin, Ch. Elster, W. N. Polyzou, W. Glc̈kle, Phys. Rev. C76(2007),014010.

4. Lin, Ch. Elster, W. N. Polyzou, W. Glëkle, Physics Letters B660(2008),345.

5. T. Lin, Ch. Elster, W. N. Polyzou, H. Witała, W. Glöckle, Phys. Rev. C78(2008)024002.

6. E. P. Wigner: Ann. Math. 40(1939)141.

7. P. A. M. Dirac, Rev. Mod. Phys. 21(1949)392.

8. P. Moussa and R. Stora in Lectures in Theoretical Physics, Vol. VIIA, Ed. W. E. Brittin and A. O. Barut, (Univeristy of Colorado Press, 1965).

9. F. Coester, Helv. Phys. Acta 38,(1965)7.

10. B.D. Keister and W. N. Polyzou, Advances in Nuclear Physics, Volume 20, Ed. J. W. Negele and E. W. Vogt, (Plenum Press 1991).

11. B. Bakamjian and L. H. Thomas, Phys. Rev. 92(1953)1300.

12. H. Ekstein, Phys. Rev. 117(1960)1590. 